\title{
A multi-wavelength view of the cosmic ray confinement in star-forming galaxies
}

\author{
P. Kornecki, ${ }^{a, *}$ E. Peretti, ${ }^{b}$ S. del Palacio, ${ }^{a}$ P. Benaglia, ${ }^{a}$ L. J. Pellizza ${ }^{c}$ and G. E. \\ Romero $^{a}$
}

${ }^{a}$ Instituto Argentino de Radioastronomía, CONICET-CICPBA-UNLP,

CC5 (1897) Villa Elisa, Prov. de Buenos Aires, Argentina,

${ }^{b}$ UNiels Bohr International Academy, Niels Bohr Institute, University of Copenhagen, Blegdamsvej 17, DK-2100 Copenhagen, Denmark

${ }^{c}$ Instituto de Astronomía y Física del Espacio, CONICET-UBA,

C.C. 67, Suc. 28, 1428 Buenos Aires, Argentina

E-mail: paulakx@iar.unlp.edu.ar, peretti@nbi.ku.dk,

sdelpalacio@iar.unlp.edu.ar, paula@iar.unlp.edu.ar, pellizza@iafe.uba.ar,

romero@iar.unlp.edu.ar

Cosmic rays (CRs) are responsible for a tight correlation between the star formation rate (SFR) and the radio/ $\gamma$-ray luminosity observed in star-forming galaxies (SFGs). This correlation can be explained by a linear scaling between the SFR and the number of CR acceleration sites, such as supernova remnants, coupled to the dependence of particle escape with galaxy properties. Observations in radio and $\gamma$-rays are important tools to probe CR activity, but they may not be sufficient to fully characterise the confinement properties of galaxies. For instance, CR calorimetry is one of the most intriguing unanswered aspects in star-forming regions that could result not only in emission through the neutrino channel but possibly also in the hard X-ray and MeV energy bands. We perform a multi-wavelength investigation of the CR population and the effective fields affecting their transport within SFGs with different levels of activity. In particular, we focus on the possibility of testing proton confinement in the $\mathrm{X}$-ray and $\mathrm{MeV}$ bands. With this goal, we develop a model describing the CR transport in SFGs for a broad range of SFRs. Hadronic byproducts, pair production and leptonic emission are computed self-consistently in a multi-wavelength context ranging from radio up to X-rays and $\gamma$-rays. We conclude that a panchromatic view of the SFRluminosity correlations in SFGs is key to place strong constraints on the physical processes that govern the non-thermal physics of these sources.

$37^{\text {th }}$ International Cosmic Ray Conference (ICRC 2021)

July 12th - 23rd, 2021

Online - Berlin, Germany

\footnotetext{
*Presenter
} 


\section{Introduction}

Galaxies with high star formation rate (SFR) are often associated to an enhanced rate of supernovae ( $\mathrm{SNe}$ ). The bulk of galactic cosmic rays (CRs) is expected to be accelerated at supernova remnant shocks [1], so that high star forming galaxies (SFGs) are naturally expected to be also powerful CR sources. In addition, the large amount of gas, photon density and the high level of turbulence produced by SNe create unique conditions for the transport of CRs in the SFG environment. CRs are, in fact, likely to lose most of their energy before being able to escape. Indeed, SFGs are known to radiate non-thermal emission in several bands of the electromagnetic spectrum from radio to $\gamma$-rays [2,3], and their observed non-thermal luminosity is in a tight correlation with the SFR. Such a correlation is expected to hold in calorimetric sources, namely in environments where most of the injected power in CRs is radiated as non-thermal photons. However, the observed correlations extend to SFGs with very low SFR, for which most of the injected particles escape their host galaxy. Investigating this phenomenology can shed light on the transport properties of electrons and protons, possibly highlighting some scaling properties ranging from the strongest calorimeters down to the most quiescent spiral galaxies.

The X-ray band is one of the most complex energy bands to decipher in SFGs. This is mainly due to the fact that their X-ray spectrum has various contributions: 1) thermal emission of the hot interstellar medium (ISM) phase; 2) point-like sources such as X-ray binaries; 3) AGN and unidentified SNRs (see e.g. [4]); 4) high-energy electrons and positrons interacting with galactic fields. In particular, the level at which high-energy particles can contribute to the X-ray flux is hard to constrain and has been poorly investigated. In the soft X-ray range $(\sim 0.2-2 \mathrm{keV})$, the emission is inferred to be dominated by the hot ISM phase and by the hot circumgalactic medium, whereas at higher energies the dominant component of the hard X-ray flux has not been clearly identified yet [19]. In the range $\sim 2-10 \mathrm{keV}$, it has been proposed that the emission in low-redshift SFGs is mainly dominated by unresolved emission from low-mass X-ray binaries. Nonetheless, the contribution from Inverse Compton (IC) scattering of galactic leptons with the infrared (IR) field can also be relevant. At higher photon energies (>30 keV) this IC emission is inferred to be the main contribution, especially in starburst galaxies [19, 20].

More recently, $[2,21,26]$ pointed out the importance of studying the X-ray band as a signature of the calorimetry level in galaxies with high SFR. In particular, in the context of individual sources, they explored the possible role played by synchrotron radiation from hadronic secondaries at 1$10 \mathrm{keV}$. In the $\mathrm{MeV}$ range the constraints on the non-thermal emission are more uncertain due to the lack of observations. In particular, the latter energy band can be of great interest for future observational campaigns and for phenomenological investigations of possible marker of presence of an non-jetted AGN in galaxies detected in $\gamma$-rays [5]. [18] explored the observed correlation between X-ray and SFR on the $0.5-8 \mathrm{keV}$ energy range, while [4] built a model to explain this X-ray luminosity-SFR correlation at higher redshift.

Here we improve upon former investigations by including more sophisticated global galaxy quantities scaling with the SFR (as the magnetic fields and the optical field from stars). We also include in our calculations the tertiary electrons, namely electron-positron pairs produced by the $\gamma-\gamma$ absorption. We discuss the diffuse hard (10-79 keV) X-ray emission and investigate the main contribution to the $\mathrm{MeV}$ energy range in SFGs. The hard X-ray band can unveil crucial aspects on 
the transport conditions of CRs in SFGs. In particular, it can be used as a unique and complementary tool to explore the confining properties of high SFGs.

\section{The emission model}

In this section we summarise the key aspects of the model, while we refer the reader to [13] and [14] for a more detailed description. The existence of the $L_{\gamma}-\mathrm{SFR}$ [6] and $L_{\text {radio }}-\mathrm{SFR}$ [8] relations suggests that a model based on the energy balance between the CR injection, escape and loss mechanisms can describe properly the global emission of SFGs.

We assume that CRs are steadily injected via diffusive shock acceleration at SNRs [e.g. 15, 16] in to the system as an exponential-cutoff power law, $Q_{i}(E)=Q_{0, i} E^{-\alpha} \exp \left[-\left(E / E_{\max , i}\right)^{\zeta_{i}}\right]$, where $i=\mathrm{e}, \mathrm{p}$ for electrons and protons, respectively, $\alpha=2.2$ is the spectral index [2], $E_{\max }$ is the maximum particle energy (assumed to be $10^{15} \mathrm{eV}$ for protons and be $10^{13} \mathrm{eV}$ for electrons), and $\zeta_{\mathrm{p}, \mathrm{e}}=(1,2)[9,10]$. For the minimum energies we adopt $E_{\mathrm{min}, \mathrm{p}}=1.2 \mathrm{GeV}$ and $E_{\mathrm{min}, \mathrm{e}}=1 \mathrm{MeV}$. We adopt $Q_{0, p} / Q_{0, e}=50[13,14,17,21]$. We normalise the accelerated proton distribution assuming that they inherit a fraction $\xi_{\mathrm{CR}}=10 \%$ of the supernova energy $\left(E_{\mathrm{SN}}=10^{51} \mathrm{erg}\right)$, so that $L_{p}=$ $\mathcal{R}_{\mathrm{SN}} E_{\mathrm{SN}} \xi_{\mathrm{SN}}$. The supernova rate of galaxies is $\mathcal{R}_{\mathrm{SN}}=\left(83 \mathrm{M}_{\odot}\right)^{-1} \dot{M}_{*}$, as prescribed by the Chabrier initial mass function [11]. Both electrons and protons experience ionisation and escape via diffusion and advection. Besides these losses, CR protons experience $p$ - $p$ interactions with the ISM, and electrons suffer IC interactions, relativistic Bremsstrahlung, and synchrotron (see [13] for the exact expressions of the corresponding timescales).

In order to compute the global emission we assume uniform injection and transport of CRs in a cylindrical volume of radius $\mathrm{R}$ and height $H=0.2 R$. We adopt $R=1 \mathrm{kpc}$, as suggested by [13]. Following [14], we describe the steady-state solution for particles in each galaxy as:

$$
N_{i}(E)=\frac{1}{|\dot{E}|} \int_{E}^{10 E_{\max , i}} Q_{i}(E) d E .
$$

Here $\dot{E}=E \tau_{\text {loss }}^{-1}(E)$ and $\tau_{\text {loss }}=1 /\left[\tau_{\text {esc }}^{-1}+\tau_{\text {cool }}^{-1}\right] ; \tau_{\text {esc }}$ is the characteristic CR escape time computed as $\tau_{\text {esc }}^{-1}=\tau_{\text {adv }}^{-1}+\tau_{\text {diff }}^{-1}$ and $\tau_{\text {cool }}^{-1}=\sum_{j} \tau_{j}^{-1}$, where $\tau_{j}$ are the CRs cooling times for the cooling mechanisms mentioned above. We finally adopt the ISM density, velocity and magnetic field scaling with the SFR described in [14] with their fiducial parameters values.

The $p-p$ interactions are responsible for the injection of secondary electrons and positrons through the decay of charged pions, in addition to $\gamma$ rays coming from the neutral pion decay. We consistently account for this secondary injection following the prescriptions of [7]. High-energy $\gamma$-ray photons, in turn, can interact with low-energy ambient photons, mainly above $\sim 10 \mathrm{TeV}$, producing electron-positron pairs. We refer to this third generation of leptons as tertiaries since the annihilating $\gamma$ rays are mostly of hadronic origin. For the $\gamma-\gamma$ interaction to occur, the energy of the $\gamma$ ray, $E_{\gamma}$, has to be above the threshold of pair production that is given by $E_{\gamma} \epsilon>\left(m_{\mathrm{e}} c^{2}\right)^{2}$, with $\epsilon$ the energy of the target photon. The optical depth $\tau_{\gamma \gamma}$ of this absorption mechanism is given by

$$
\tau_{\gamma \gamma}=2 H \int_{m_{\mathrm{e}}^{2} c^{4} / E_{\gamma}}^{\infty} \sigma_{\gamma \gamma}\left(E_{\gamma}, \epsilon\right) n_{\mathrm{ph}}(\epsilon) d \epsilon
$$

where $\sigma_{\gamma \gamma}\left(E_{\gamma}, \epsilon\right)$ is the total cross section of the interaction assuming an isotopic target photon field with number energy distribution $n_{\mathrm{ph}}(\epsilon)$ [12]. The relevant target photon fields are the UV 

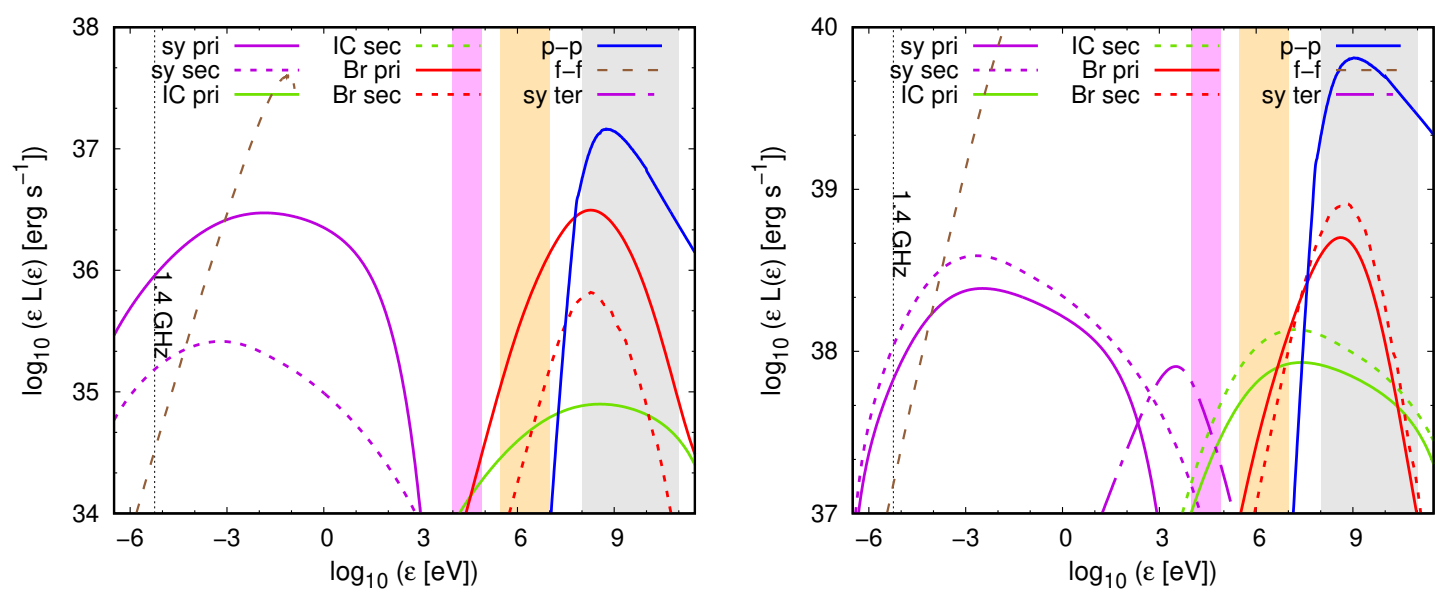

Figure 1: Example SEDs computed for the benchmark scenario of [14]. The left panel corresponds to a low SFR $\left(\dot{M}_{*}=0.1 M_{\odot} / \mathrm{yr}\right)$ and the right panel to a high SFR $\left(\dot{M}_{*}=10 M_{\odot} / \mathrm{yr}\right)$. The coloured lines (solid for the primary CRs, short-dashed for the secondary and dashed-dot for tertiary) are the individual contributions of different emission processes. The dot-dashed brown line is the thermal free-free emission. The grey, orange and magenta shaded regions represent the Fermi-LAT, e-ASTROGAM and NuSTAR energy ranges, respectively, and the vertical dotted black line shows the studied radio frequency of $1.4 \mathrm{GHz}$.

starlight reprocessed in IR and the optical unabsorbed starlight. The CMB photon field has a small density and its effects on electron cooling and $\gamma$-ray absorption within the galaxies are negligible.

Assuming that the $\gamma-\gamma$ absorption is taking place in the leading particle regime, namely when all energy is transferred to one electron, the rate of tertiary injection can be calculated using the approximation given by [12],

$$
Q_{\mathrm{ter}}\left(E_{e}\right)=2 H \int_{m_{\mathrm{e}}^{2} c^{4} / E_{\gamma}}^{\infty} Q_{\gamma}\left(E_{e}\right) \sigma_{\gamma \gamma}\left(E_{e}, \epsilon\right) n_{\mathrm{ph}}(\epsilon) d \epsilon=\tilde{\tau}_{\gamma \gamma} Q_{\gamma}\left(E_{e}\right)
$$

where $Q_{\gamma}\left(E_{e}\right)$ is the $\gamma$-ray photon emissivity multiplied by the galaxy volume and $\tilde{\tau}_{\gamma \gamma}=\min \left[1, \tau_{\gamma \gamma}\right]$.

\section{Multi-wavelength view on the SFR-Luminosity correlation}

We solve Eq. 1 and compute the energy distribution of all particle generations using the SFR as a unique independent variable. We then compute the broadband spectral energy distribution (SED) in the whole SFR range. We show in Fig. 1 the SED for two archetypal cases of low $\left(0.1 \mathrm{M}_{\odot} \mathrm{yr}^{-1}\right)$ and high $\left(10 \mathrm{M}_{\odot} \mathrm{yr}^{-1}\right)$ SFRs. Below we provide an overview of the main physical processes that govern the SED in different energy bands and discuss how observations in each specific band can help to improve our knowledge of the non thermal properties of SFGs.

Radio: The emission in the frequency range $10-100 \mathrm{GHz}$ is mainly due to free-free emission, making the exploration of the non-thermal component challenging. On the contrary, the lowfrequency $(v \lesssim 10 \mathrm{GHz}$ ) radio domain is typically dominated by synchrotron radiation produced by relativistic electrons. This emission depends on the total power in relativistic electrons and the intensity of the ambient magnetic fields. In particular, in low-SFR galaxies, the major contribution 
to the radio emission comes from primary electrons whose energy distribution is dominated by escape. The contribution of synchrotron emission from secondaries increases its relative importance compared to the contribution from primaries for higher SFRs (see left panel of Fig. 1).

In addition, for higher SFRs the higher ISM densities lead to a more significant role of ionisation and bremsstrahlung losses. The prospects of investigating sources at frequencies $v<1 \mathrm{GHz}$ are bright thanks to radio interferometers such as LOFAR [22] and the uGMRT [23]. This frequency range has the advantage of minimising the thermal contamination, though the impact of absorption processes can highly reduce the outgoing fluxes. Nonetheless, exploring the absorption features could provide indications on the density of the ionised material (and thus of the ionisation rate [14]).

X-rays: The study of the diffuse, non-thermal emission produced by CRs is more suitable to be performed in the hard X-rays $(>10 \mathrm{keV})$, where the thermal contamination is likely subdominant. The NUSTAR satellite is currently observing the sky in the 3-79 keV energy range [25] and can provide unique information on the transport conditions of both electrons and protons. For low-SFR galaxies, the production of hard X-ray emission is inefficient and limited to relativistic bremsstrahlung and IC of primary electrons. However, for high-SFR galaxies, the IC emission from secondary electrons increases its relevance and dominates the SED (see Fig. 1). Moreover the stronger IR field typical of high SFR leads to a more efficient absorption of $\gamma$ rays, which leads to efficient production of tertiary leptons (with electron energies $\approx 50 \mathrm{TeV}$ ). Interestingly, secondary and tertiary electrons are responsible for a strong emission of synchrotron radiation in the $\sim 10 \mathrm{keV}$ range. Therefore, the intensity of the hard $\mathrm{X}$-ray emission reflects both the magnetic field intensity and the calorimetry level of the parent protons.

MeV $\gamma$ rays: This energy range has been poorly explored so far. However, missions currently planned such as $e$-ASTROGAM have the potential to open a window for novel studies in the $0.3-$ $10 \mathrm{MeV}$ energy range [24]. The continuum emission here can only be of non-thermal origin. We show in Fig. 1 that bremsstrahlung produced by primary leptons is dominant in galaxies with low SFR, whereas the IC emission dominate in the case of high SFR. In particular, the IC emission is dominated by secondary leptons for higher SFRs. Leptons radiating IC are cooled due to both IC and synchrotron. According to our model, an exposure time of several years would be required in order to detect the nearest SBGs in this energy band with e-ASTROGAM. An earlier detection would imply either a weaker magnetic field or the presence of additional sources in the galaxy (such as an AGN or a superposition of several mild point-like sources).

GeV $\gamma$ rays: A breakthrough in the $\mathrm{GeV}$ energy range was provided by the Fermi-LAT observatory which has detected 14 SFGs so far [13]. The GeV $\gamma$ ray emission is dominated by he neutral pion decay resulting from $p$ - $p$ inelastic collisions. For high SFRs, the protons responsible for this emission are in a calorimeter regime and only $p-p$ interactions are relevant. For lower SFRs, however, escape processes (advective or diffusive) can be important and significantly reduce the radiative output.

SFR-Luminosity correlations: We calculated the integrated luminosity in the energy bands of Fermi-LAT $\left(L_{\gamma}\right)$ and e-ASTROGAM $\left(L_{\mathrm{MeV}}\right)$, and in the hard X-ray energy range accessible to 

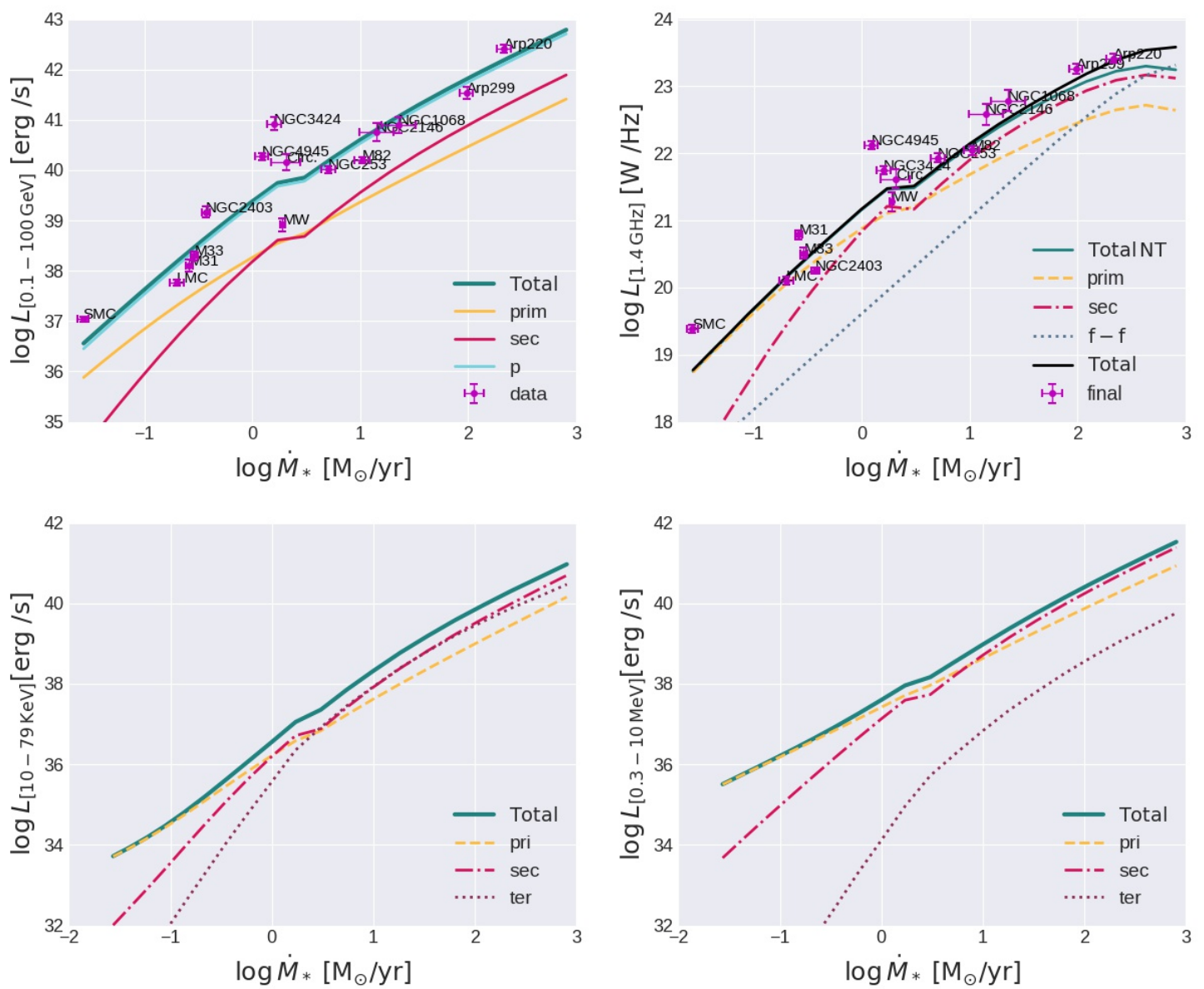

Figure 2: Modelled correlations computed from the benchmark case of [14]. In each case the yellow dashed, the red dot-dashed and the dotted purple lines represent the primary, secondary and tertiary electron contribution, respectively. The solid green line represents the total non-thermal emission. In the top panels the magenta points indicate the data sample from [14]. Top left panel: The $L_{\gamma}-\mathrm{SFR}$ relation. Top right panel: The $L_{1.4 \mathrm{GHz}}-\mathrm{SFR}$ relation. The dotted green line is the free-free contribution and the solid black line is total emission (thermal + non-thermal). Bottom left panel: $L_{\mathrm{X}}-\mathrm{SFR}$ relation. Bottom right panel: $L_{\mathrm{MeV}}-\mathrm{SFR}$ relation.

NuSTAR (10-79 keV; $\left.L_{\mathrm{X}}\right)$. We also computed the radio luminosity at $1.4 \mathrm{GHz}\left(L_{1.4 \mathrm{GHz}}\right)$. We show in Fig. 2 the correlations $L_{\gamma}-\mathrm{SFR}, L_{\mathrm{MeV}}-\mathrm{SFR}, L_{\mathrm{X}}-\mathrm{SFR}$ and $L_{1.4 \mathrm{GHz}}-\mathrm{SFR}$. In each case we show the relative contribution of the total emission from each generation of particles, namely protons (p) and primary (prim), secondary (sec) and tertiary (ter) electrons. We also report the contribution of thermal free-free (f-f) in the radio-SFR correlation.

The $L_{\gamma}-\mathrm{SFR}$ correlation is dominated by $p-p$ emission. For $\dot{M}_{*}>30 \mathrm{M}_{\odot} \mathrm{yr}^{-1}$ the correlation slope becomes quasi-linear indicating that the model reached the calorimetric limit. At low SFRs, protons are efficiently escaping from the emission region, and as a consequence the correlation departs from the calorimetric regime and the correlation slope steepens. The $L_{1.4 \mathrm{GHz}}-\mathrm{SFR}$ at low SFR $\left(\dot{M}_{*}<5 \mathrm{M}_{\odot} \mathrm{yr}^{-1}\right)$ is shaped by the synchrotron emission of primary electrons, although these particles can also diffuse away from the emission region. At higher SFRs sources become electron 
calorimeters and secondary electrons dominate the non-thermal emission. Such an important contribution from secondaries highlights the transition to a calorimetric regime for protons in SFGs. In particular, the emission of secondary electrons becomes dominant for $\dot{M}_{*}>30 M_{\odot} \mathrm{yr}^{-1}$.

Bremsstrahlung and IC from primary electrons dominate the $L_{X}-$ SFR correlation at low SFRs, while for $\dot{M}$ in the range $5-30 \mathrm{M}_{\odot} /$ yr the synchrotron from secondaries and tertiaries dominates. For higher SFRs the IC from secondaries overcomes the synchrotron.

The density of tertiary electrons does not only depend on the IR field, but it is also directly related to the emissivity of $\gamma$ rays, which in turn depends on the level of calorimetry of protons. Hence, modulo the magnetic field strength that could be estimated by scaling relations and radio observations, an enhanced hard X-ray flux could be adopted to explore proton calorimetry in SFGs being a smoking gun of high synchrotron emission from both secondaries and tertiaries and IC from secondaries.

The $L_{\mathrm{MeV}}-\mathrm{SFR}$ correlation shows that bremsstrahlung from primary leptons is dominant for low SFRs, whereas for $\dot{M}>5 \mathrm{M}_{\odot} /$ yr the IC emission becomes more relevant. In particular the higher the SFR the higher the relative contribution of secondaries. Since electrons cool efficiently via synchrotron, the amount of $\mathrm{MeV} \gamma$ rays produced also depends on the magnetic field intensity, being smaller for higher magnetic fields.

\section{Summary and final remarks}

We presented an emission model for SFGs valid over a broad range of SFRs. We explored how the transport of CRs accelerated in a star-forming environment shapes the $L_{\gamma}-\mathrm{SFR}, L_{1.4 \mathrm{GHz}}-\mathrm{SFR}$, $L_{\mathrm{X}}-\mathrm{SFR}$ and $L_{\mathrm{MeV}}-\mathrm{SFR}$ correlations for SFGs. We also compared our modelled correlation with the most updated observational data available in the $1.4 \mathrm{GHz}$ and $\gamma$-ray energies. From the radio and $\gamma$-ray correlations we learnt that galaxies with star formation rate greater than $\sim 30 \mathrm{M}_{\odot} / \mathrm{yr}$ are proton and electron calorimeters. The coexistence of synchrotron from primary and secondary electrons is essential to reproduce the observed $L_{1.4 \mathrm{GHz}}-\mathrm{SFR}$ correlation. The X-ray band has an important contribution from synchrotron emission produced by tertiaries in addition to the emission from secondaries. In particular, the strong synchrotron emission from both secondaries and tertiaries could provide new indications and constraints on both magnetic fields and calorimetry in galaxies with high SFR. The emission in the MeV energy band can be quite sensitive to the magnetic field intensity as well. The parameter space that defines the correlations in the hard X-ray and $\mathrm{MeV}$ bands together with the possible observations will be studied in greater details in a forthcoming work. By doing so, we aim at further breaking the degeneracy between the free/unknown parameters by pursuing this multi-wavelength approach to the SFR-Luminosity correlations.

\section{References}

[1] Blasi, P. 2013, A\&ARev, 21, 70. doi:10.1007/s00159-013-0070-7

[2] Lacki, B. C. \& Thompson, T. A. 2013, ApJ, 762, 29. doi:10.1088/0004-637X/762/1/29

[3] Romero, G. E., et al. 2018, A\&A, 616, A57. doi:10.1051/0004-6361/201832666 
[4] Schober, J., et al. 2015, MNRAS, 446, 2. doi:10.1093/mnras/stu1999

[5] Padovani, P. 2017, Frontiers in Astr. \& Space Sci., 4, 35. doi:10.3389/fspas.2017.00035

[6] Ackermann, M., et al. 2012, ApJ, 755, 164. doi:10.1088/0004-637X/755/2/164

[7] Kelner, S. R., et al. 2006, PhRvD, 74, 034018. doi:10.1103/PhysRevD.74.034018

[8] Bell, E. F. 2003, ApJ, 586, 794. doi:10.1086/367829

[9] Zirakashvili, V. N. \& Aharonian, F. 2007, A\&A, 465, 695. doi:10.1051/0004-6361:20066494

[10] Blasi, P. 2010, MNRAS, 402, 2807. doi:10.1111/j.1365-2966.2009.16110.x

[11] Chabrier, G. 2003, PASP, 115, 763. doi:10.1086/376392

[12] Aharonian, F., Bergström, L., \& Dermer, C. 2013, Astrophysics at Very High Energies,Springer-Verlag Berlin Heidelberg, 2013, 40. doi:10.1007/978-3-642-36134-07

[13] Kornecki, P., et al. 2020, A\&A, 641, A147. doi:10.1051/0004-6361/202038428

[14] Kornecki, P., et al. 2021, arXiv:2107.00823 [astro-ph.HE]

[15] Axford, W. I., Leer, E., \& Skadron, G. 1977, International Cosmic Ray Conference, 11, 132

[16] Bell, A. R. 1978, MNRAS, 182, 147. doi:10.1093/mnras/182.2.147

[17] Yoast-Hull, T. M., et al. 2013, ApJ, 768, 53. doi:10.1088/0004-637X/768/1/53

[18] Sarkar, K. C., et al. 2016, ApJ, 818, L24. doi:10.3847/2041-8205/818/2/L24

[19] Wik, D. R., et al. 2014, ApJ, 797, 79. doi:10.1088/0004-637X/797/2/79

[20] Persic, M. \& Rephaeli, Y. 2002, A\&A, 382, 843. doi:10.1051/0004-6361:20011679

[21] Peretti, E., et al. 2019, 36th International Cosmic Ray Conference (ICRC2019), 36, 382

[22] van Haarlem, M. P., et al. 2013, A\&A, 556, A2. doi:10.1051/0004-6361/201220873

[23] Gupta, Y., Ajithkumar, B., Kale, H. S., et al. 2017, Current Science, 113, 707

[24] de Angelis, A., et al. 2018, Journal of High Energy Astrophysics, 19, 1. doi:10.1016/j.jheap.2018.07.001

[25] Harrison, F. A., et al. 2013, ApJ, 770, 103. doi:10.1088/0004-637X/770/2/103

[26] Peretti E., et al., 2019, MNRAS, 487, 168. doi:10.1093/mnras/stz1161 